\title{
Towards unraveling the moisture-induced shape memory effect of wood: the role of interface mechanics revealed by upscaling atomistic to composite modeling
}

\author{
Chi Zhang $\mathbb{1}^{1}$, Mingyang Chen ${ }^{1}$, Sinan Keten ${ }^{2}$, Dominique Derome ${ }^{3}$ and Jan Carmeliet ${ }^{1}$
}

\begin{abstract}
The moisture-induced shape memory effect (SME) is one of the most intriguing phenomena of wood, where wood can stably retain a certain deformed shape and, upon moisture sorption, can recover the original shape. Despite the long history of wood utilization, the SME is still not fully understood. Combining molecular dynamics (MD) and finiteelement (FE) modeling, a possible mechanism of the SME of wood cell walls is explored, emphasizing the role of interface mechanics, a factor previously overlooked. Interface mechanics extracted from molecular simulations are implemented in different mechanical models solved by FEs, representing three configurations encountered in wood cell walls. These models incorporate moisture-dependent elastic moduli of the matrix and moisture-dependent behavior of the interface. One configuration, denoted as a mechanical hotspot with a fiber-fiber interface, is found to particularly strengthen the SME. Systematic parametric studies reveal that interface mechanics could be the source of shape memory. Notably, upon wetting, the interface is weak and soft, and the material can be easily deformed. Upon drying, the interface becomes strong and stiff, and composite deformation can be locked. When the interface is wetted again and weakened, the previously locked deformation cannot be sustained, and recovery occurs. The elastic energy and topological information stored in the cellulose fiber network is the driving force of the recovery process. This work proposes an interface behaving as a moisture-induced molecular switch.
\end{abstract}

\section{Introduction}

The shape memory effect (SME) of wood, also referred to as hygrolock-springback ${ }^{1,2}$ or set-recovery ${ }^{3}$, is one of the most intriguing mechanical effects. Wood, after deformation in the wet state and being dried under maintained deformation, retains the deformed shape even after the removal of the mechanical loading, denoted as fixation. Wood recovers the original shape upon subsequent wetting, denoted as recovery. Wood fixation is

Correspondence: Chi Zhang (zhangchi@ethz.ch)

'Department of Mechanical and Process Engineering, ETH Zurich, 8092 Zurich, Switzerland

${ }^{2}$ Department of Civil and Environmental Engineering, Northwestern University, 2145 Sheridan Road, Evanston, IL 60208-3109, USA

Full list of author information is available at the end of the article frequently sought, for example, to obtain shape stability of bentwood in curved furniture. In contrast, the recovery tendency may pose difficulties, for example, in the case of densified wood, which may recover a certain volume upon water adsorption and experience weakening ${ }^{4}$. A better understanding of the mechanisms of the SME can be helpful in inspiring the design and fabrication of natureinspired smart materials, e.g., cell-bundle torsional actuators with memory ${ }^{5}$, and improvement of the stability of deformed wood ${ }^{4}$.

This study focuses on the moisture-induced SME at the wood cell wall level. The reasoning is described as follows: the SME can be observed at different scales under varying conditions. At the timber level, the SME has been attributed to the multiscale hierarchical structure of wood

\section{(c) The Author(s) 2021}

(c) (i) Open Access This article is licensed under a Creative Commons Attribution 4.0 International License, which permits use, sharing, adaptation, distribution and reproduction cc) in any medium or format, as long as you give appropriate credit to the original author(s) and the source, provide a link to the Creative Commons license, and indicate if changes were made. The images or other third party material in this article are included in the article's Creative Commons license, unless indicated otherwise in a credit line to the material. If material is not included in the article's Creative Commons license and your intended use is not permitted by statutory regulation or exceeds the permitted use, you will need to obtain permission directly from the copyright holder. To view a copy of this license, visit http://creativecommons.org/licenses/by/4.0/. 
tissue $^{6}$. However, increasing evidence suggests that it is highly possible that the SME of wood originates from the cell wall material. For example, Plaza et al. ${ }^{5}$ observed a memory effect in nanoindentation, where an indented dry middle lamella fully recovered upon wetting with a drop of water. In another example, Derome et al. ${ }^{7}$ observed half-cell moisture-induced shape recovery after a cell was mechanically deformed. To date, the microscopic mechanism of wood cell wall-level SMEs has rarely been mentioned in previous studies. The SME can be triggered by various stimuli, such as temperature ${ }^{6}$, moisture ${ }^{6}$, and temperature-moisture combined ${ }^{8,9}$. It is noted that a high temperature may induce a series of chemical reactions, e.g., pyrolysis, which is beyond the scope of this study. Instead, this study focuses on the moisture-induced SME.

It is imperative to differentiate the SME from the shape change effect (SCE). The SCE is a common phenomenon of almost all materials. Materials deform in response to environmental stimuli, such as heat, mechanical loading, magnetic field, light, and moisture, and materials revert to their original shape after loading removal. A typical example of the SCE is the hygroscopic swelling of wood, where wood expands due to the adsorption of water and shrinks with desorption. This reversible swelling and shrinking feature of plant cell walls is the source of several natural actuation mechanisms that facilitate processes in plant life cycles, such as the release of seeds from pine cones upon humidity changes ${ }^{10}$ or the self-planting mechanism of wheat seeds ${ }^{11}$. In contrast to the SCE, the SME indicates that the deformation of materials is maintained even after the removal of environmental stimuli and that the original shape is recovered only when certain conditions are satisfied. The SME is similar to the SCE in the sense that the affected materials finally recover their original shapes, whereas deformation maintenance in an intermediate state is the main characteristic of the SME.

Although the SME of wood has been described in numerous reports, the microscopic mechanisms remain unexplained. The vast majority of available literature has covered either practical solutions to reduce springback of densified wood or phenomenological descriptions of the macroscopic behavior of wood, leaving the microscopic mechanism of the moisture-induced SME of wood cell walls barely elucidated. In one of the few examples, Colmars developed a one-dimensional discrete formulation of a hygrolock model describing the mechanical behavior with humidity cycles ${ }^{12}$ and found that extra high creep occurs in dry wood, which could be attributed to the presence of high internal stresses.

The mechanism of the polymer SME seems well established. Two submechanisms are essential ${ }^{13,14}: 1$. potential energy or topological information is stored via chemical or physical crosslinking bonds; 2. a molecular switch kinetically controls two states via glass transition, (re)crystallization, etc. Although wood cell walls are in principle polymer composites, to date, only speculations or hypotheses regarding the question of which wood microstructures are responsible for these submechanisms have been reported. As the SME likely originates from molecular interactions at the nanometer scale, a major difficulty lies in the experimental challenges faced when gathering high-resolution information on these sophisticated hydrated biopolymer composite structures. Atomistic numerical studies are thus warranted as a complement to experimental investigations to consider which nanoscale wood features could be involved in the SME.

The shape memory behavior of wood involves multiple mechanical and sorption loading/unloading steps. Thus, the origin of the SME behavior of this complex material can involve multiple sources. We considered several possibilities. In the first hypothesis, swelling-induced deformation and alteration of the hydrogen-bonded state are suspected to be at the origin. When dried in the deformed state, reformation of hydrogen bonds results in material stiffening and deformation maintenance. When wetted again, the structure swells, possibly driving shape recovery. One critical missing point is the agent of memory. While the shape changes in response to wetting, how is the structure recovery direction determined? Information on the initial shape is somehow stored which is yet to be explained ${ }^{9}$. In the second hypothesis, glass transition is suspected to play a role in structure switching between rigid and flexible states, but a possible explanation of how information on the initial shape is stored is not provided. Third, the mechanosorptive effect, i.e., the coupling between deformation and sorption, was considered. This has been an active research area recently ${ }^{15}$. Mechanosorptive creep could explain the retention of the deformed shape but does not explain the subsequent recovery. These considerations lead to the conclusion that the matrix alone cannot fully explain the SME. Cellulose fibers should thus be considered. Recent observations of the plant cell wall suggest that the mainly crystalline cellulose (CC) fibers come into contact at certain positions, referred to as hotspots, thus forming possible connected fiber meshes ${ }^{16-19}$. These fiber contacts can be considered a type of interface formed between the fibers, while the fiber-matrix interface prevails in the cell wall material. The co-occurrence of two different interfaces has been largely overlooked and might play a role in the SME. In this study, we analyze whether the occurrence and configuration of interfaces between fiber-matrix and between fiber-fiber, and their dependence on moisture comprise a system functioning as the necessary molecular switch between fixation and recovery. 
In this study, finite-element (FE) models of prototype wood cell wall layers are built. The hygromechanical properties of the interfaces are obtained via atomistic simulations, mainly molecular dynamics (MD) simulations, while the other mechanical parameters needed for FE model construction are retrieved from atomistic works in the literature. A loading protocol, applied to evaluate the moisture-induced SME, questions two main aspects: whether deformation imposed under wet conditions can be sustained under dry conditions and whether the original shape can be recovered when wetted again.

\section{Materials and methods}

Three systems, representing possible configurations of the $\mathrm{S} 2$ cell wall, are simulated with continuum mechanics. The computational domains, material properties, boundary conditions, and loading protocol are presented.

\section{Geometry of the model}

The wood cell wall S2 layer features a complex hierarchical ultrastructure for which a definitive understanding of its material arrangement has not yet been attained $^{20}$. The S2 layer, the thickest layer of the cell wall, is commonly treated as a unidirectional fiber-reinforced composite with CC fibers aligned at an angle, referred to as the microfibril angle (MFA), to the axial direction of the cell, as illustrated in Fig. 1a. Studies have assumed that hemicellulose chains connect cellulose fibers, forming the so-called tethered network ${ }^{21,22}$. Recent observations of the plant cell wall suggest that the abovementioned fibers are not perfectly parallel. In contrast, they may come into contact either directly or mediated by a monolayer of hemicellulose $\mathrm{e}^{16-19}$. The contacts between fibers, in addition to the resultant connected network structure, have been referred to as mechanical hotspots, structures that remain to be investigated.

It should be noted that the S2 material is different between the three directions, i.e., radial (R), tangential (Ta), and longitudinal (L). The currently widely accepted model is the so-called concentric lamella model, suggesting that $\mathrm{S} 2$ comprises concentric rings, layer upon layer, as shown in Fig. 1a. The building elements of these concentric lamella are cellulose aggregates, with a thickness of $\sim 25 \mathrm{~nm}$, formed by microfibrils $\sim 3-4 \mathrm{~nm}$ in diameter $^{23-25}$. In the radial direction, the connection between lamellae is weak. It can be assumed that mechanical hotspots may only be present in the tangential plane, while in the radial plane, cellulose fibers mainly remain parallel, as shown in Fig. 1b. Considering these assumptions, three two-dimensional subunits are considered basic building elements of the S2 layer, as shown in the dashed square, triangular, and circular regions in Fig. 1b, covering all possible scenarios. The subunit marked with a square includes cellulose fibers surrounded by the matrix. The subunit marked with a triangle indicates two fibers in direct contact surrounded by the matrix. The subunit marked with a circle is similar to the triangular subunit, except that the fibers are not in perfect contact and are only in contact over a finite length. These subunits are applied as representative two-dimensional geometries, namely, Models $1-3$, respectively, as shown in Fig. 1c. For simplicity, only 2D models are considered assuming plane stress conditions. The interfaces and materials are represented with lines and bulk colors, respectively. Models 1 and 2 are parallel combinations, but in Model 3, the fibers form an undulating pattern, where the axial direction remains parallel to the domain edges. In the following, the longitudinal (L) and transverse $(\mathrm{R} / \mathrm{Ta})$ directions are referred to as the $x$ and $y$ directions, respectively. In terms of polymers, the fibers comprise CC. For the sake of simplicity, the matrix is assumed to consist only of hemicellulose galactoglucomannan (GGM), as experimental results have suggested that GGM is the matrix material most adjacent to $\mathrm{CC}$ fibers in $\operatorname{wood}^{26}$.

In terms of the full geometry, Models 1-3, as shown in Fig. 1c, are replicated three times along both the $x$ and $y$ directions to reduce edge effects. The resulting geometries are shown in Fig. $1 \mathrm{~d}$. The total length and height are $l=$ $72 \mathrm{~nm}$ and $h=36 \mathrm{~nm}$, respectively. The length is chosen

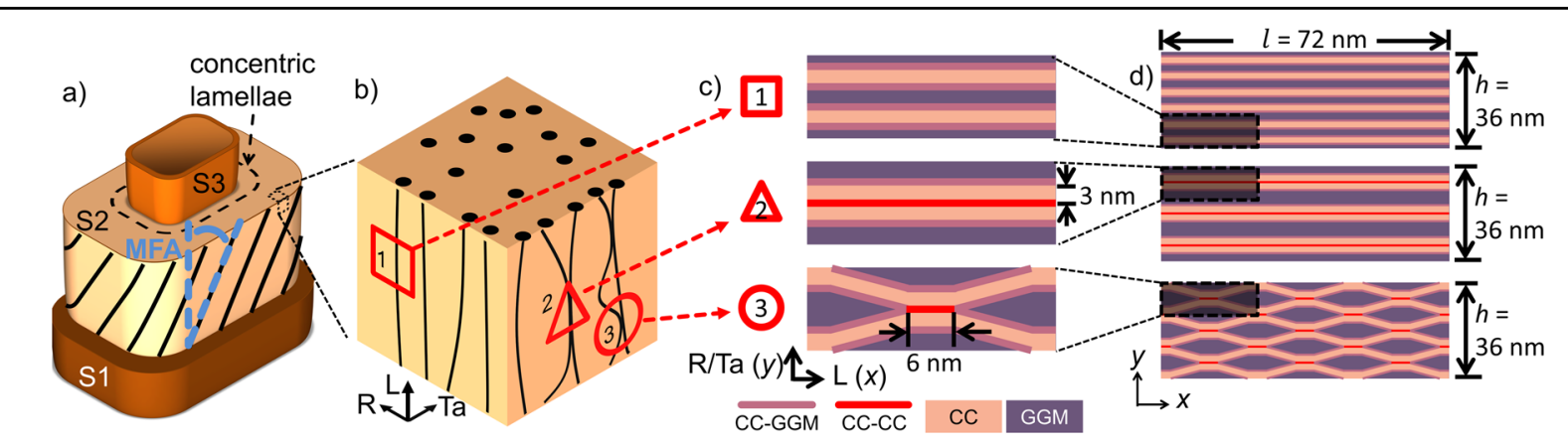

Fig. 1 Schematic material arrangements in wood cell wall. a secondary cell wall layers with $\mathbf{b}$ close-up of S2. 
to closely reflect the experimentally reported cellulose nanocrystal length of $\sim 100 \mathrm{~nm}^{27}$. The height in principle can be any arbitrary value, but here, the height is chosen to include three repetitions along the $y$ direction. As is shown below, the resulting specimen size is sufficiently large to simulate the SME effect but is sufficiently small from a computational cost point of view.

To facilitate comparison, the models are constructed as similar as possible. The mass ratio of fibers to the matrix is $1: 1$, in line with experimental reports ${ }^{28}$. The three models are of the same length and height. The cellulose fibers are $3 \mathrm{~nm}$ in width ${ }^{28}$. The CC-CC contact length in Model 3 is assumed to be $6 \mathrm{~nm}^{29}$. Details on the meshing of Model 1 are included in Supporting Information Fig. S1 as an example. In general, the meshes are refined at the interfaces or boundaries and coarser in the bulk region, and a mesh sensitivity analysis is carried out.

These models represent three basic probable configurations of the above assumed representative volume element of the wood cell wall layer. Much care is taken toward appropriate implementation of the swelling behavior of the matrix and moisture weakening phenomenon of the matrix and interfaces, as described in the following section.

\section{Constitutive laws of the components and interfaces}

Modeling wood cell wall material can be overwhelmingly complicated because of the multiple components and hierarchical structure, in addition to the ratedependent response of mechanical loading and coupled deformation-sorption behavior. For the sake of feasibility, this study is limited to the hygroelastic response involving stick-slip behavior at the interfaces. Moisture-related mechanical features of the components and interfaces are obtained based on MD. Comprehensive validations are performed to ensure the validity of the molecular models against experimental data, such as density, modulus, and swelling coefficient, the details of which can be found in our previous reports ${ }^{30-36}$.

As presented above, the FE models contain two materials, namely, CC and GGM, and two interfaces, namely, CC-GGM and CC-CC. These two materials are modeled with a linear-elastic material model and hygroscopic swelling, as described in the following equation:

$$
\varepsilon=\varepsilon_{\text {elastic }}+\varepsilon_{\text {hygroscopic swelling }}=C \sigma+\beta m
$$

where $\varepsilon, C, \sigma, \beta$ and $m$ are the strain, compliance, stress, hygroscopic swelling coefficient and moisture content, respectively. The mechanical properties of $\mathrm{CC}$ are independent of moisture, as suggested in many experimental and simulation studies ${ }^{37,38}$, and the hygroscopic swelling coefficient is set to 0 . The stiffness of $\mathrm{CC}$ along the $\mathrm{R}$ and Ta directions varies considerably (at 11.3 and $72.6 \mathrm{GPa}$, respectively ${ }^{39}$ ). Since this study is $2 \mathrm{D}$ research, $\mathrm{CC}$ is assumed to be a transversely isotropic material, and the average stiffness along the $\mathrm{R}$ and Ta directions is adopted as the stiffness along the transverse direction $(\mathrm{T})$. The elastic behavior of transversely isotropic fibers is described by five mechanical properties, as retrieved from the literature ${ }^{39-42}$ and listed in Table 1. GGM is an isotropic material that interacts with moisture and, therefore, can be represented by Young's and shear moduli and swelling coefficient based on MD measurements in previous work of our research group ${ }^{36}$, as summarized in Table 1.

Based on the elastic properties in Table 1, the other elastic properties of isotropic materials can be calculated with the following equations according to continuum mechanics:

$$
K=\frac{E G}{3(3 G-E)} \text { and } \nu=\frac{E}{2 G}-1
$$

In regard to a transversely isotropic material, the following equations apply:

$$
\frac{\nu_{\mathrm{LT}}}{E_{\mathrm{L}}}=\frac{\nu_{\mathrm{TL}}}{E_{\mathrm{T}}} \text { and } G_{\mathrm{TL}}=G_{\mathrm{LT}}
$$

There are two types of interfaces between the materials, namely, the $\mathrm{CC}-\mathrm{CC}$ and $\mathrm{CC}-\mathrm{GGM}$ interfaces. The moisture-dependent interface properties and shear strength as a function of the moisture content, as summarized in Table 2, are extracted from the results of pulling tests carried out via atomistic modeling, as described in refs. ${ }^{30,43}$. The shear strength is found to linearly correlate with the interfacial hydrogen bond density. At the atomistic scale, hydrogen bonds are formed between hydrogen donors and acceptors, and both are negatively charged oxygen atoms. Hydrogen bonding is stronger than other nonbonded interactions

\begin{tabular}{|c|c|c|c|c|c|c|}
\hline \multirow[b]{2}{*}{$\mathrm{CC}$} & \multicolumn{2}{|c|}{ Young's modulus (GPa) } & \multirow{2}{*}{$\begin{array}{l}\text { Shear modulus (GPa) } \\
G_{L T}=4.4\end{array}$} & \multicolumn{2}{|c|}{ Poisson's ratio } & \multirow{2}{*}{$\begin{array}{l}\text { Swelling coefficient } \\
\beta=0\end{array}$} \\
\hline & $E_{\mathrm{L}}=150$ & $E_{\mathrm{T}}=42$ & & $v_{L T}=0.38$ & $v_{\pi T}=0.48$ & \\
\hline GGM & \multicolumn{2}{|c|}{$E(m)=5.4 \exp (-7.2 m)$} & $G(m)=1.8 \exp (-5.7 m)$ & - & & $\beta=0.49$ \\
\hline
\end{tabular}
and plays a pivotal role in the mechanics of biomolecules

Table 1 Elastic properties of CC and GGM G6,39-42 $^{3}$ 
Table 2 Interface laws $s^{30,43}$.

\begin{tabular}{lll}
\hline & Parallel direction & Perpendicular direction \\
\hline CC-GGM & $\tau_{\max }(\mathrm{m})=0.11 \exp (-1.15 \mathrm{~m})$ & Non-separation \\
CC-CC & $\tau_{\max }(\mathrm{m})=0.45 \exp (-3.95 \mathrm{~m})$ & \\
\hline
\end{tabular}

such as proteins, DNA, and cellulose fiber. Hydrogen bonds constitute a microscopic feature present in large quantities, and these bonds strongly influence the macroscopic mechanical behavior of wood cell wall components. When moisture is adsorbed at the interface, the hydrogen bond network is partly broken, resulting in interface weakening and shear strength reduction. The interface subjected to shear undergoes a typical stick-slip behavior with a sawtooth profile in stress-displacement curves. This study assumes a simplified interface model where the interface sticks when the shear stress is below the friction threshold equal to the maximum shear stress. Sliding occurs when the shear stress at the interface equals the maximum shear stress. With increasing sliding, the shear stress remains constant and equal to the maximum shear stress. The maximum shear stress depends on the moisture level. Friction is assumed to be independent of the normal pressure ${ }^{15}$. Sliding along the direction parallel to the interface is permitted, but separation along the direction perpendicular to the interface is not allowed.

These interface laws are implemented using contact elements in the FE modeling. The adopted FE software is Ansys Mechanical APDL 16.2. The bulk material is modeled with the PLANE182 element. The contact elements are TARGE169 and CONTA171.

\section{Boundary conditions and loading protocol}

The three models bear the same boundary conditions and loading protocol. In wood steam bending in practice, a form is employed. Inspired by this practice, the bending model used is shown in Fig. 2a, where the specimen is mounted atop a cylindrical support and a displacement or load is applied on the ends ${ }^{6}$. There exists a symmetry plane, and therefore, only half of the beam model is considered as the computational domain to reduce the computational cost. This setup is modeled with the FEM, and it is found that the results of this setup correspond to the results of the FE model under the given boundary conditions, as shown in Fig. 2b. A roller constraint is applied on the left edge, allowing only transverse displacement. The left bottom node is fixed, which is also adopted as the origin of the coordinate system. It is noted that cylinder support is also tested, and the results are identical to the point support results. A vertical point load is applied by imposing a displacement $d$ along the $y$-axis on the free right top node. Moisture affects the system via the constitutive equations of the materials and interfaces, causing swelling and weakening of GGM and weakening of the interfaces.

Based on experimental studies demonstrating the recovery of half-cell walls from the deformed state ${ }^{7}$ and the macroscopic timber $\mathrm{SME}^{6}$, a loading protocol is proposed. The mechanical and moisture loading protocol can be summarized as a 7-step procedure: initial state with null moisture and mechanical loading $(\mathrm{N})$, uniform wet state (W), mechanical loading under wet conditions imposing displacement (WS), dry state while maintaining displacement $(\mathrm{S})$, displacement removal $\left(\mathrm{N}^{\prime}\right)$, wet state again $\left(\mathrm{W}^{\prime}\right)$ and dry state again $\left(\mathrm{N}^{\prime \prime}\right)$. The 7 steps are illustrated in Fig. 2c, d. In the wet states, i.e., W, WS and $\mathrm{W}^{\prime}$, the moisture content in the system is assumed to be uniform and equal to $m=0.3$. In the mechanically loaded states, i.e., WS and $\mathrm{S}$, the vertical displacement applied is $d=-7.2 \mathrm{~nm}$, corresponding to a deflection ratio of $\gamma=d /$ $l=0.1$. The deflection ratio in experimental shape memory studies is usually high, e.g., close to $1^{6}$. However, here, a lower ratio value is applied to improve convergence and maintain material deformation within the elastic range. The $\mathrm{N}, \mathrm{N}^{\prime}$, and $\mathrm{N}^{\prime \prime}$ states correspond to the initial, deformed, and final states, respectively. The $\mathrm{S}$ state is the state with the largest deformation.

\section{Results \\ SME with the three models}

The three models, representing possible wood cell wall material arrangements, are subjected to the 7-step loading protocol. Qualitatively similar behaviors are observed in all the systems. Since Model 3 reveals the most evident shape memory, i.e., fixation and recovery, it is discussed first.

Snapshots of Model 3 are shown in Fig. 3. The color denotes the normal stress $\sigma_{x}$ (Fig. 3a) and shear stress $\tau_{x y}$ (Fig. 3b). The displacement is magnified by a factor of 2 for the sake of clarity. The dashed line indicates the $y=0$ plane. A magnified view of the center region is shown in Fig. S2.

The models are subjected to the proposed 7-step loading protocol, comprising states $\mathrm{N}, \mathrm{W}, \mathrm{WS}, \mathrm{S}, \mathrm{N}^{\prime}, \mathrm{W}^{\prime}$, and $\mathrm{N}^{\prime \prime}$, as described in the "Materials and methods" section. In state $\mathrm{W}$, the matrix swells due to moisture sorption, while the CC phase does not. Swelling generates internal stresses, i.e., tensile stress in the CC phase and compressive stress in the GGM matrix, with shear stresses in the material and at the interfaces. In the WS state, the normal tensile stresses increase in the top region, while compressive normal stresses arise at the bottom due to bending. The shear stresses also increase at the CC-GGM and $\mathrm{CC}-\mathrm{CC}$ interfaces, reaching maximal shear stress values at certain locations where sliding occurs. $\mathrm{CC}-\mathrm{GGM}$ is a weaker interface than is $\mathrm{CC}-\mathrm{CC}$, therefore 

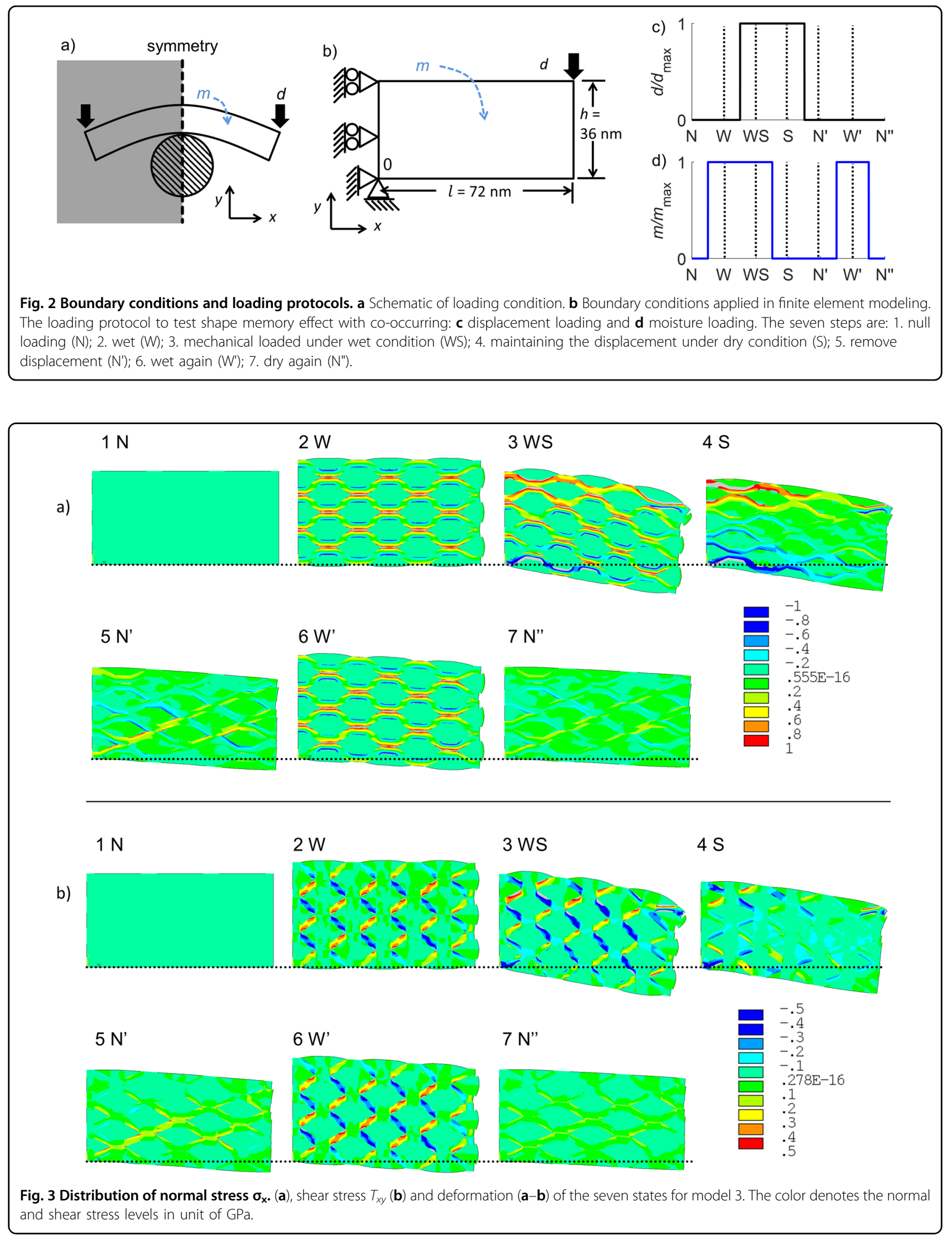


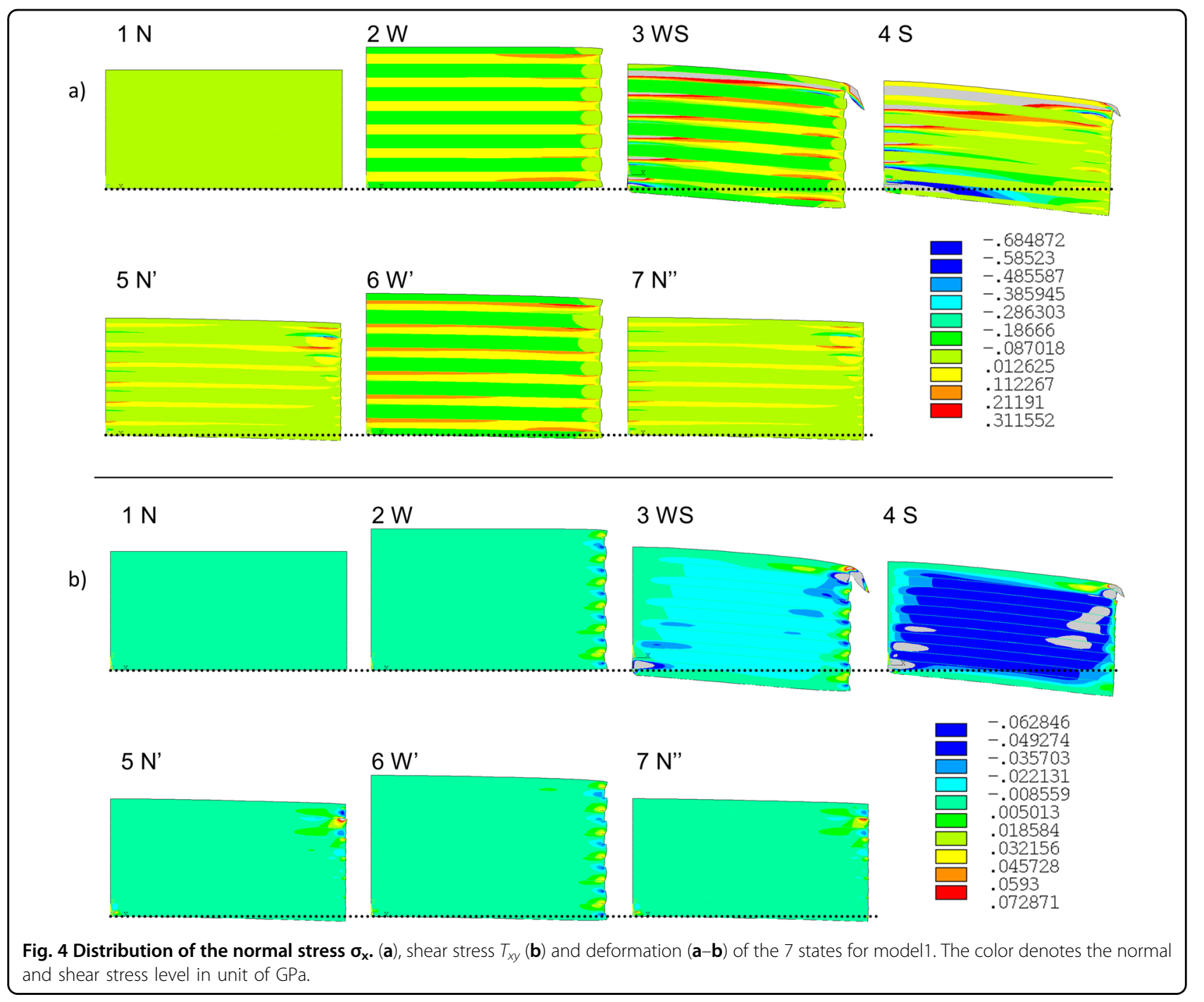

sliding commences at the CC-GGM interface, followed by sliding at select $\mathrm{CC}-\mathrm{CC}$ hotspots. Upon drying to reach the $\mathrm{S}$ state, the matrix shrinks, and the shear stresses decrease due to a reduction in differential deformation between the matrix and CC phase. Upon unloading to attain state $\mathrm{N}^{\prime}$, residual shear stresses and deformation remain in the specimen, indicating that the specimen partially maintains its deformed state, referred to as fixation. When the specimen is wetted thereafter (W '), the matrix swells again, and differential stresses between CC and the matrix again occur, but it is observed that the vertical deformation decreases since slip behavior may occur at the interfaces. The decrease in deformation after wetting is referred to as recovery. Finally, when the specimen is dried again $\left(\mathrm{N}^{\prime \prime}\right)$, residual stresses and permanent deformation may remain in the specimen.

Snapshots of Models 1 and 2 are shown in Figs. 4 and 5, respectively. Recall that Model 1 comprises several alternating layers of CC and GGM with CC-GGM interfaces. Model 2 also consists of layers of $\mathrm{CC}$ and GGM, with both CC-CC and CC-GGM interfaces. Models 1 and 2 achieve similar behavior to that of Model 3 across the seven states. However, the above laminated structures are much softer than is Model 3 with an undulating fiber mesh. As a result, the point loads in Models 1 and 2 are lower than those in Model 3 for the same deflection. Consequently, the normal and shear stresses in Models 1 and 2 are much lower than those in Model 3. Due to the lower shear stresses at the interfaces, less sliding occurs in Models 1 and 2. This indicates that Model 3 with a stiff undulating fiber mesh containing $\mathrm{CC}$ hotspots promotes interface sliding and, as is shown below, leads to the most notable SME, while the SME is not as evident in Models 1 and 2. The gray areas in Figs. 4 and 5 denote high-stress zones. 


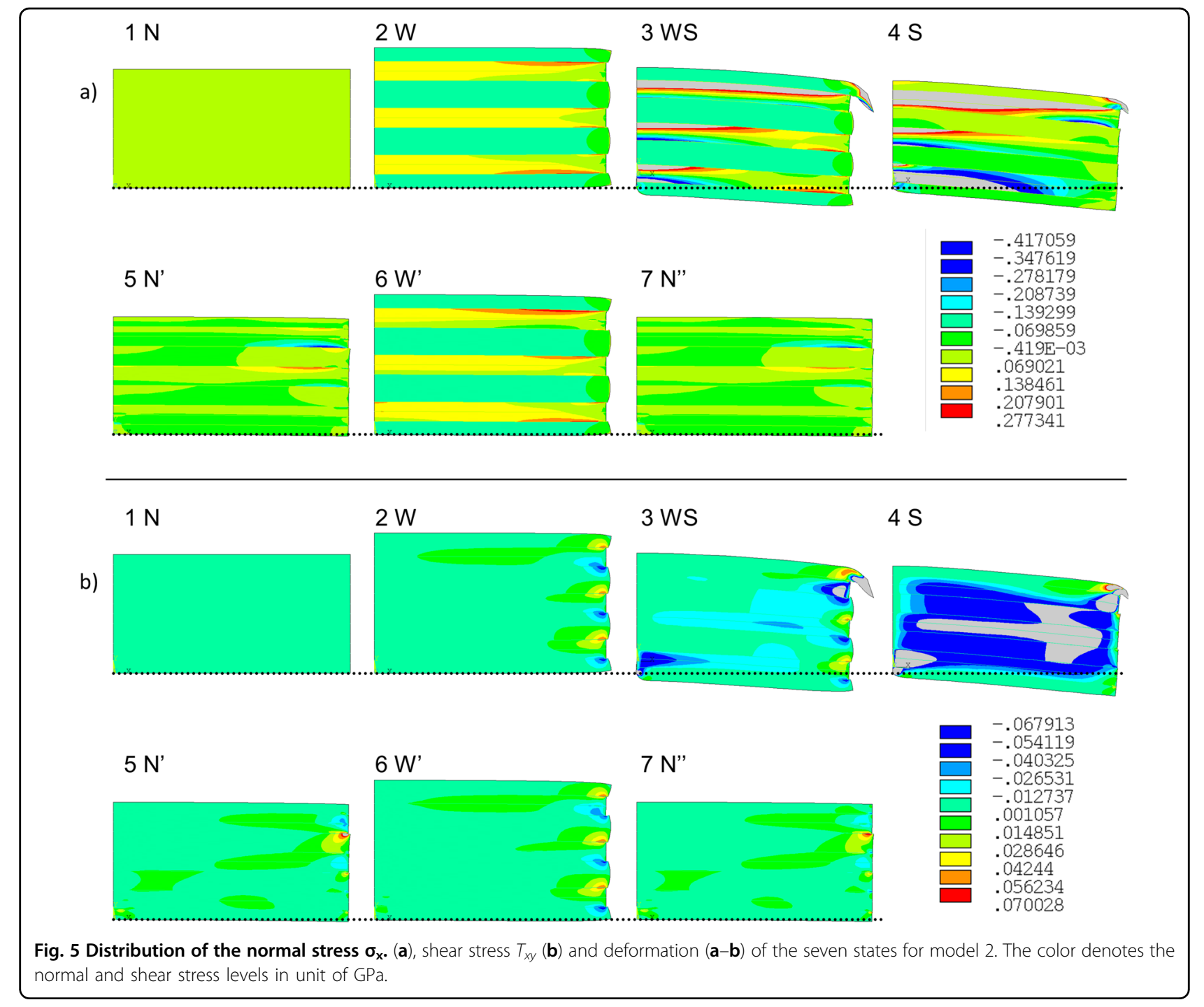

Deformation is quantified by the deflection ratio $\gamma$ defined as the ratio of $y$-direction displacement $u_{y}$ to length $l$ :

$$
\gamma=\frac{u_{y}}{l}
$$

It is noted that $y$-direction displacement $u_{y}$ is the difference in $y$-direction displacement between $x=0 \mathrm{~nm}$ and $x=$ $72 \mathrm{~nm}$. The deflection ratio is averaged along the right outer edge at $x=72 \mathrm{~nm}$. As $u_{y}$ attains a negative value, the absolute values of $\gamma$ are considered below for the sake of convenience.

The shape fixation behavior can be identified by comparing the deformation levels between states $\mathrm{S}$ and $\mathrm{N}^{\prime}$. Upon deformation under mechanical loading in the wet state, the specimen is dried, reaching state $\mathrm{S}$ characterized by $\left|\gamma_{\mathrm{S}}\right|$. After removal of the mechanical load, state $\mathrm{N}^{\prime}$ is reached, as characterized by $\left|\gamma_{\mathrm{N}^{\prime}}\right|$. In state $\mathrm{N}^{\prime}$, three possible scenarios apply:
$\left|\gamma_{\mathrm{N}^{\prime}}\right|=\left|\gamma_{\mathrm{S}}\right|,\left|\gamma_{\mathrm{S}}\right|>\left|\gamma_{\mathrm{N}^{\prime}}\right|>0$ and $\left|\gamma_{\mathrm{N}^{\prime}}\right|=0$. For $\left|\gamma_{\mathrm{N}^{\prime}}\right|=\left|\gamma_{\mathrm{S}}\right|$, the material maintains the applied deformation, referred to as perfect fixation. For $\left|\gamma_{S}\right|>\left|\gamma_{\mathrm{N}^{\prime}}\right|>0$, the material reveals permanent intermediary deformation, referred to as partial fixation. However, in the case of $\left|\gamma_{\mathrm{N}^{\prime}}\right|=0$, the material returns to the initial state, indicating no fixation nor shape memory. The deflection ratio $\gamma$ of the three models over the specimen length in the $\mathrm{S}, \mathrm{N}^{\prime}$ and $\mathrm{N}^{\prime \prime}$ states is shown in Fig. 6. The three models all demonstrate partial fixation, i.e., $\left|\gamma_{\mathrm{S}}\right|>\left|\gamma_{\mathrm{N}^{\prime}}\right|>0$. This suggests that the deformed shape in the $\mathrm{S}$ state is partially maintained, which is in accordance with previous experimental observations ${ }^{6}$. The difference between $\left|\gamma_{\mathrm{N}^{\prime}}\right|$ and $\left|\gamma_{\mathrm{S}}\right|$ is attributed to the decrease in elastic deformation after specimen drying and mechanical loading removal.

The shape recovery behavior can be identified by comparing the deformation levels between states $\mathrm{N}^{\prime}$ and $\mathrm{N}^{\prime \prime}$. Starting from the $\mathrm{N}^{\prime}$ state, the system is again wetted and 

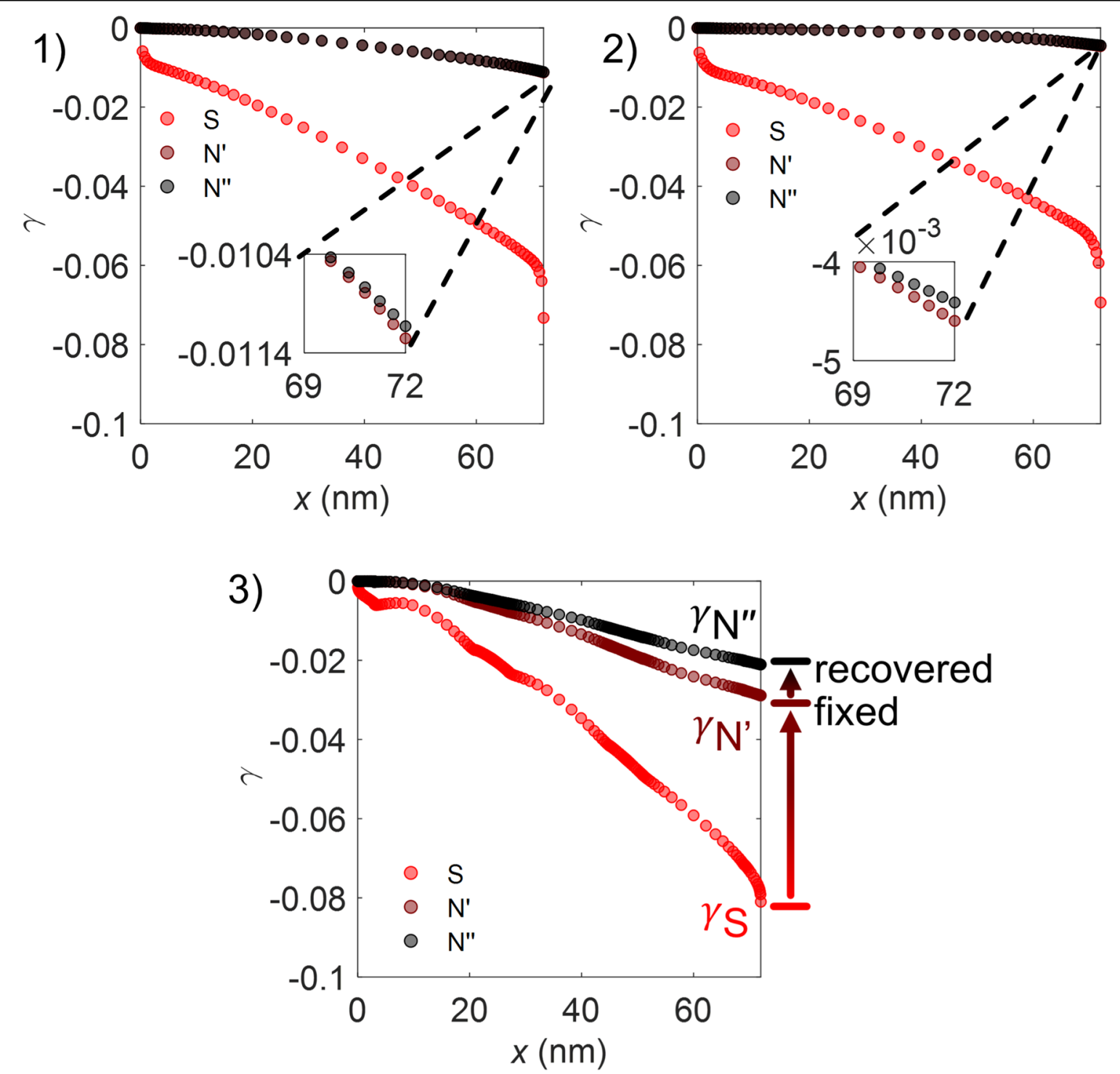

Fig. 6 Deflection ratio of the models 1, 2 and 3 over the length of the specimen at states $S, N^{\prime}$ and N".

dried, reaching the final state $\mathrm{N}^{\prime \prime}$. The deflection ratio $\left|\gamma_{\mathrm{N}^{\prime \prime}}\right|$ varies between 0 and $\left|\gamma_{\mathrm{N}^{\prime}}\right|$. For $\left|\gamma_{\mathrm{N}^{\prime \prime}}\right|=\left|\gamma_{\mathrm{N}^{\prime}}\right|$, the material retains its deformation associated with the $\mathrm{N}^{\prime}$ state, indicating that no recovery occurs. The other extreme, i.e., $\left|\gamma_{\mathrm{N}^{\prime \prime}}\right|=0$, indicates full recovery to the initial state, also referred to as total shape recovery. All three systems reveal recovery behavior, although the recovery of Models 1 and 2 is negligible, as shown in the insets of Fig. 6.

The fixation behavior can be quantified by the fixation ratio $R_{\mathrm{f}}$, defined as ${ }^{44}$

$$
R_{\mathrm{f}}=\frac{\gamma_{\mathrm{N}^{\prime}}-\gamma_{\mathrm{N}}}{\gamma_{\mathrm{S}}-\gamma_{\mathrm{N}}}
$$

In effect, this quantity represents the deformation amount maintained in intermediate state $\mathrm{N}^{\prime}$ over deformed state $\mathrm{S}$. It should be noted that, in our study, the initial state $\gamma_{N}=0$. The recovery ratio is similarly defined, representing the deformation amount maintained in the final $\mathrm{N}^{\prime \prime}$ state over the deformed $\mathrm{S}$ state:

$$
R_{\mathrm{r}}=\frac{\gamma_{\mathrm{N}^{\prime \prime}}-\gamma_{\mathrm{N}}}{\gamma_{\mathrm{S}}-\gamma_{\mathrm{N}}}
$$

A fixation ratio of 1 suggests full fixation, and a recovery ratio of 0 suggests full recovery. Conversely, a fixation ratio of 0 indicates no fixation, and a recovery ratio of 1 
Table 3 Summary of the models: deflection, fixation and recovery ratios.

\begin{tabular}{lllllll}
\hline Model & $\left|\gamma_{\mathbf{N}}\right|$ & $\left|\gamma_{\mathbf{S}}\right|$ & $\left|\gamma_{\mathbf{N}^{\prime}}\right|$ & $\left|\gamma_{\mathbf{N}^{\prime \prime}}\right|$ & $\boldsymbol{R}_{\mathbf{f}}$ & $\boldsymbol{R}_{\mathbf{r}}$ \\
\hline 1 & 0 & 0.073 & 0.0112 & 0.0116 & 0.154 & 0.152 \\
2 & 0 & 0.069 & 0.005 & 0.004 & 0.066 & 0.064 \\
3 & 0 & 0.081 & 0.029 & 0.021 & 0.356 & 0.260 \\
\hline
\end{tabular}

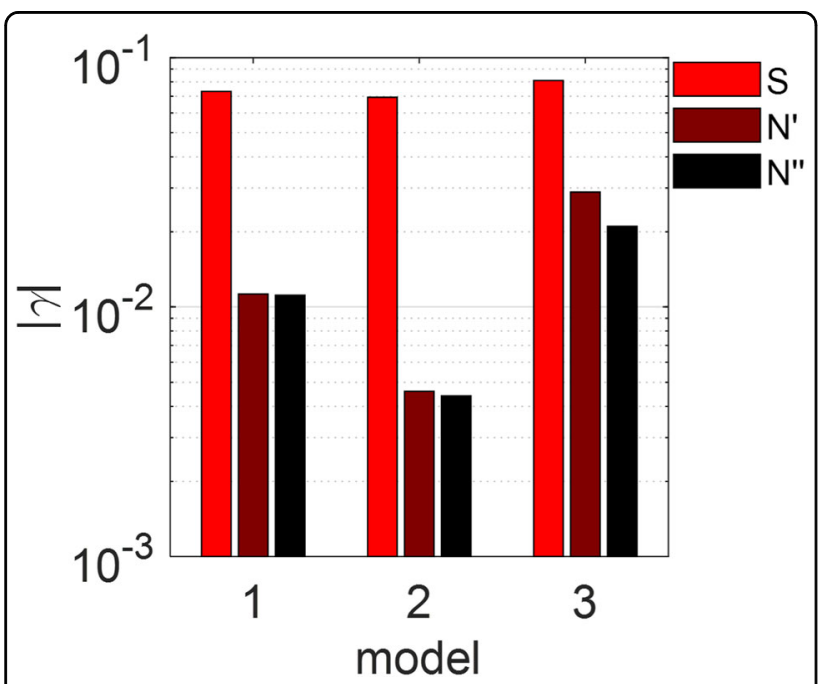

Fig. 7 Deflection ratio at the right edge for models 1,2 and 3 in states $\mathrm{S}, \mathrm{N}^{\prime}$ and $\mathrm{N}^{\prime \prime}$.

indicates no recovery. When the fixation and recovery ratios are nonzero and equal values, there is no recovery and almost no SME is observed.

The deflection, fixation and recovery ratios of the three models, considered at the right edge, $x=72 \mathrm{~nm}$, are listed in Table 3. Model 3 attains the highest fixation and recovery ratios, and Model 2 attains the lowest fixation and recovery ratios. It should be noted that the fixation and recovery ratios are almost equal between Models 1 and 2 .

It is necessary to understand why Model 3 yields a more evident SME. The deflection ratio at the right edge $x=72 \mathrm{~nm}$ in the $\mathrm{S}, \mathrm{N}^{\prime}$, and $\mathrm{N}^{\prime \prime}$ states is shown in Fig. 7 . This figure exhibits a semilog scale. The magnitude of the deformation in state $\mathrm{S}$ (red color) is similar across all models. The fixation in state $\mathrm{N}^{\prime}$ (brown color) is the highest for Model 3, while for Model 2, it is the smallest. For Model 3, the fibers undulate and form connected networks with $\mathrm{CC}-\mathrm{CC}$ hotspots, leading to a much stiffer structure than that of Models 1 and 2. Consequently, the mechanical load and shear stresses in Model 3 are higher, leading to much greater sliding at the CC-GGM interfaces. Then, the $\mathrm{CC}-\mathrm{CC}$ contacts act as zones constraining system deformation, and this behavior prevents springback and contributes to locking of the deformed shape.

\section{Origin of the SME: interface-controlled fixation and recovery mechanisms}

Moisture induces three changes in the system, namely, swelling of the matrix, weakening of the matrix and weakening of the interfaces. To study the effect of these three moisture influences on the SME, we activated and deactivated these moisture dependencies by modifying the constitutive relationship. To omit matrix swelling, the swelling coefficient of the matrix can be set to 0 . To omit weakening of the matrix and interfaces, the parameter $m$ in the corresponding controlling functions can be set to 0 . In total, $2^{3}=8$ different cases can be generated based on these three effects. The conclusion is that fixation and recovery are only possible when introducing moisturedependent interface mechanics, while moisture-induced swelling and weakening of the matrix are not sufficient to generate the SME.

Here, we include one of the 8 systems, referred to as Model 4, built by deactivating the moisture dependency of the interfaces in Model 3. The fixation ratio of Model 4 is zero, indicating that after the removal of external displacement, the system fully regains its original shape. There is no fixation, leading to the absence of SME. This indicates that interface weakening and strengthening, the so-called molecular switch, is the source of the moisture-induced SME. The molecular-level mechanism of interface switching between hydrogen-bonded and nonhydrogen-bonded states controls the fixation and recovery phenomena of the system.

The size of the moisture-induced molecular switch can be assessed by determining the sliding distance. Among the four models, the average sliding distance $d_{\text {slid. }}$ of the two interfaces of CC-GGM and CC-CC (except Model 1) are shown in Fig. 8a. Model 3 achieves much larger sliding distances than do the other models. Model 1 attains small sliding distances at the CC-GGM interfaces, while Model 2 indicates that sliding mainly occurs at the $\mathrm{CC}-\mathrm{CC}$ interfaces. All models exhibit permanent sliding in the final $\mathrm{N}^{\prime \prime}$ state, suggesting that there is no full backslip and thus no full recovery.

In state $\mathrm{N}^{\prime}$, the interface occurs in the strong dry state and is prevented from slipping back at the interfaces, which further prevents the springback phenomenon of the specimen and explains the resulting fixation. During specimen wetting (the $\mathrm{N}^{\prime}-\mathrm{W}^{\prime}-\mathrm{N}^{\prime \prime}$ steps), the interface is weakened by moisture and can slide back, releasing a portion of the deformation until the point where the shear stress attains equilibrium with friction, as provided by the wet weak interface. The elastic energy stored in the material is the driving force of the recovery process. 


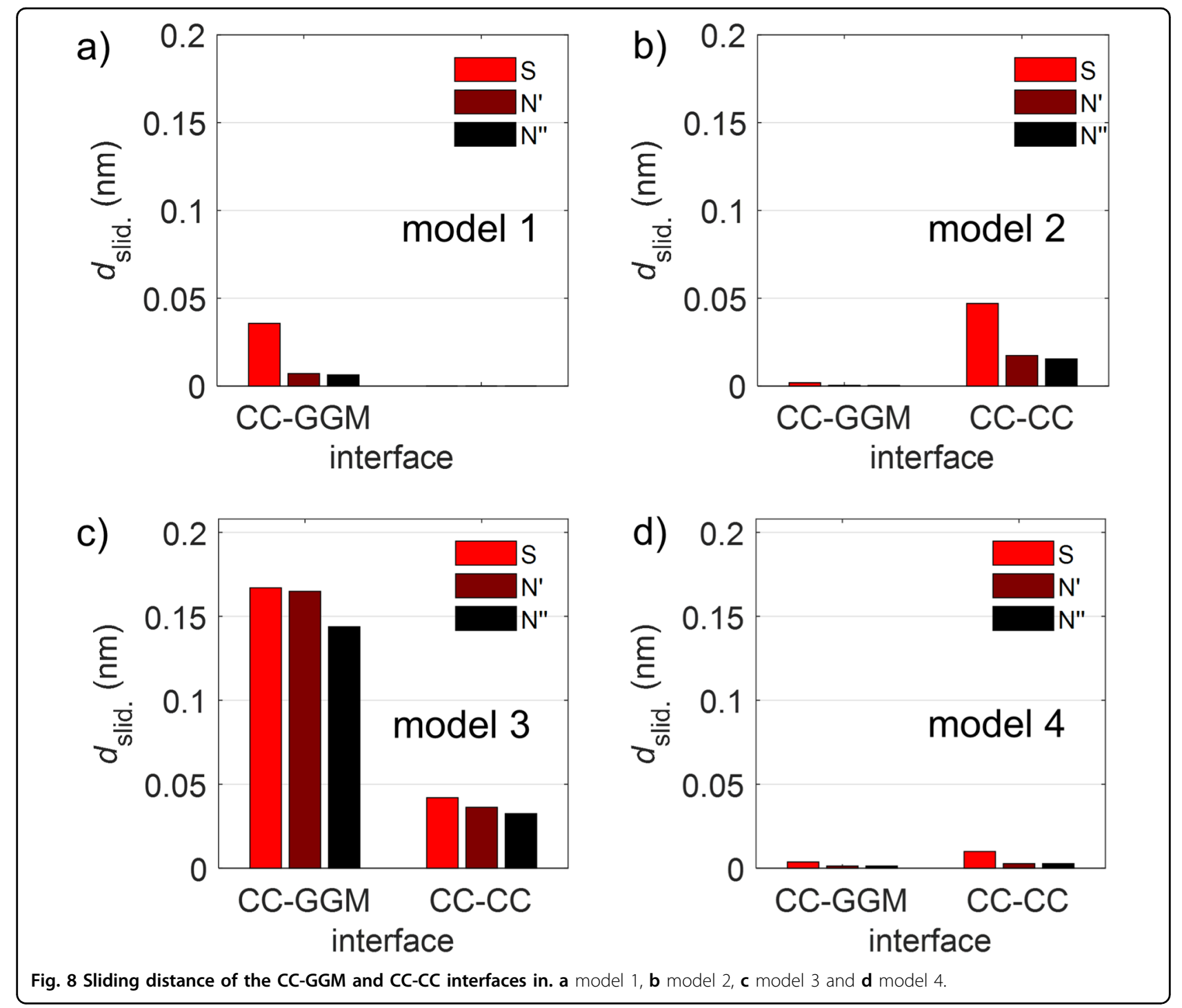

Following the discussion above, the moisture-induced SME mechanism can be summarized as follows and is shown in Fig. 9, capturing the evolution of the deflection ratio $\gamma$ versus the moisture content:

1. N, which is the initial state without deformation and stress. The interfaces are strong, achieved by abundant hydrogen bonding. The molecular switch is not active, and no sliding is allowed.

2. W, the material is wetted and swells. Hydrogen bonds are broken, thereby weakening the interfaces. The molecular switch is active, i.e., deformation is likely to occur.

3. WS, a vertical mechanical load is applied to the wet material. In addition to mainly elastic deformation, components slide against each other at the interfaces, thereby forming a new material configuration.

4. S, moisture is removed with the applied mechanical loading maintained. Upon water removal, hydrogen bonds are reformed across the interfaces, thus strengthening these interfaces. The molecular switch is not active, hence locking the material configuration.

5. $\mathrm{N}^{\prime}$, the external loading is removed. Most of the deformation is maintained by the locking effect of the interfaces, referred to as fixation. A portion of the deformation is lost due to the release of elastic energy. The shear stress at the interface occurs in equilibrium with the strength of the dry interface.

6. $W^{\prime}$, the material is wetted again. The interface is weakened. Thus, the material loses its ability to maintain the deformed shape, and recovery occurs. The deformation retained in state $\mathrm{N}^{\prime}$ decreases, as the non-negligible friction provided by the weak interface prevents full recovery. The shear stress at the interface is reduced to match the strength level of the wet interface.

7. $\mathrm{N}^{\prime \prime}$, the material is dried again. Compared to the initial state $\mathrm{N}$, residual deformation is observed. 


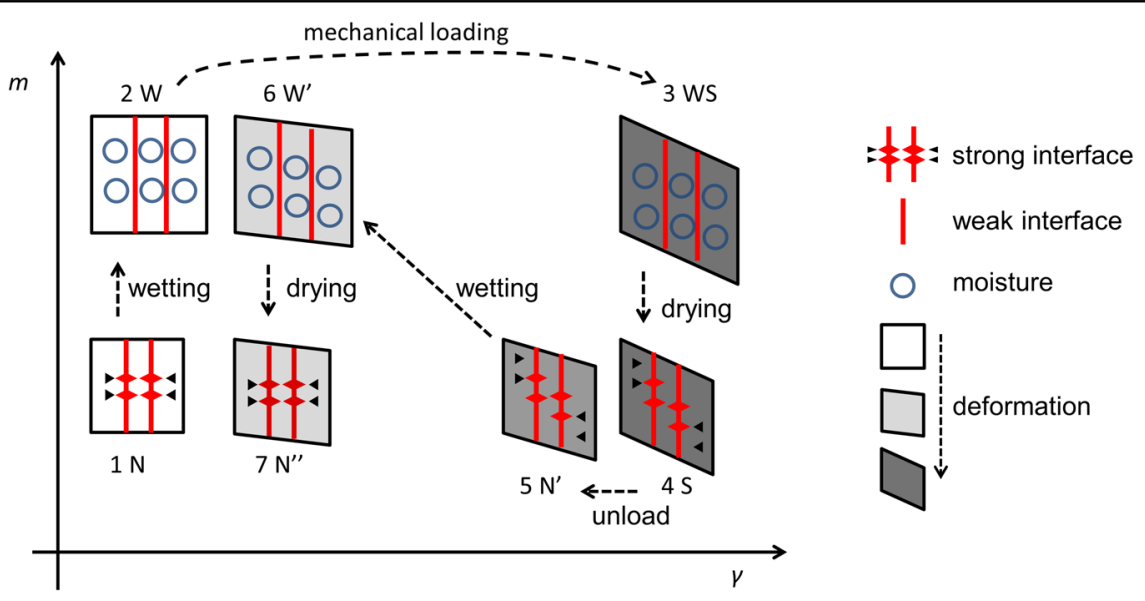

Fig. 9 Schematic of the moisture-induced shape memory effect.

\section{Discussion}

As shown above, interface mechanics are at the core of the SME. Elaborating on the interpretation, a more abstract and conceptual representation of the SME can be elucidated in terms of an energy landscape, as shown in Fig. S3. The potential energy of the system is denoted as $\Gamma$, which is a function of the location of the system in the phase space denoted by a variable $q\left(\gamma, d_{\text {slid. }}\right)$, which combines the influence of $\gamma$, the deflection ratio, and $d_{\text {slid, }}$, the sliding distance. Therefore, the state of the specimen is characterized by its loading state $q$ and energy state $\Gamma$. The system starts in the initial $\mathrm{N}$ state located at the minimum of the dry potential surface. When wetting occurs to reach state $\mathrm{W}$, the molecular switch is active, and the system moves from the dry potential surface to the minimum of the wet potential surface. When mechanically loaded, the system moves away from the minimum of the wet potential energy surface to state WS. Upon drying to attain state $\mathrm{S}$, the system moves from the wet potential surface to the dry potential energy surface, remaining at a high energy level since the applied mechanical loading is maintained. When mechanically unloaded to reach state $\mathrm{N}^{\prime}$, the system follows the dry potential energy surface to its minimum, remaining at a certain potential energy level due to fixation, i.e., with the molecular switch inactive. Upon wetting, i.e., as the molecular switch is activated, to reach state $\mathrm{W}^{\prime}$, the system again moves to the wet potential energy surface, but energy is lost and the system moves down along the wet energy surface. It should be noted that state $\mathrm{W}^{\prime}$ is located at higher $q$ and $\Gamma$ levels above state $\mathrm{N}$, indicating that some deformation and energy remain stored in the system. Finally, the system is dried to reach state $\mathrm{N}^{\prime \prime}$. In summary, these two energy minima correspond to the fixed and recovered states, while moisture acts as the activator. Through the wet potential energy surface, the system can migrate from a surface with a high potential energy to a low-energy surface.
For any material exhibiting an SME, there should be at least two local energy minima/metastable states. Under activation by external stimuli, e.g., moisture in this case, the energy barrier between these two states is lowered, kinetically locked potential energy is released, and the system reaches a more thermodynamically stable state.

The SME of wood entails a complex phenomenon involving multiple scales and a wide range of physical and mechanical sub-mechanisms. This study presents a possible mechanism of the moisture-induced SME intrinsic to wood cell wall composite materials. In contrast to most existing studies, where the SME is attributed to bulk properties, such as crystallization, glass transition, and chemical bonding, this study aims to demonstrate that interface mechanics play an important role, a largely overlooked factor. It is demonstrated that with moisturedependent interface mechanics, the material can exhibit the SME, including fixation and recovery, and removal of the interface moisture dependency eliminates SME occurrence. It should be noted that moisture-induced interface weakening does not encompass a glass transition-like process, a phenomenon normally defined as the process whereby a supercooled melt yields, upon cooling, a glassy structure ${ }^{45}$.

Notably, this study is focused on a possible mechanism of the SME of wood cell wall material. To date, the finestscale experiment involved a half-cell study ${ }^{7}$, where both the cell wall material and cellular structure exhibited shape memory. Since wood mostly comprises cell walls, it is highly likely that the macroscopic wood behavior also originates at the cell wall material level. As verified in the half-cell study, the recovery process of the macroscopic cellulose structure shape can be regarded as a result of the deformed cell wall regaining its initial shape. To further demonstrate this finding, it is feasible that, in a future study, a macroscopic wood cellular structure can be 
modeled with shape memory behavior embedded in the constitutive cell wall material laws.

Although the composite material in this study can capture the major moisture-related behavior aspects of cell wall composite materials, it differs from realistic cell wall materials in several aspects: 1 . the considered materials include CC and GGM, whereas the other hemicelluloses and lignins are not included. 2. The materials are assumed to act elastically, which might be valid under small deformation at short timescales. Based on these considerations, future studies can be expected to include the following features: 1 . more components of the cell wall should be introduced into the FE model; 2. nonlinear material laws should be considered, such as mechanosorptive effects described by poromechanics ${ }^{15,46}$, and time-dependent viscoelastic effects and moisture transport through diffusion should be accounted for. 3. Three-dimensional models that may bestow a more obvious SME should be developed.

In conclusion, this study probes one of the possible mechanisms of the SME of wood cell wall composite materials, i.e., a moisture-controlled molecular switch based on interface mechanics. The shape memory of wood may have multiscale origins, but few studies have been conducted at the cell wall level. Prototypical cell wall systems propose a mechanism of the SME that is dominated by the stick-slip behavior of interfaces: first, breakage and reformation of hydrogen bonds at the fiber-matrix interface serve as a moisture-induced molecular switch responsible for shape fixation and recovery. Second, the elastic energy stored in the fibril functions as the driving force of shape recovery. To achieve these findings, this study considers the co-occurrence of two different interfaces under specific configurations.

Atomistic insights into interface mechanics obtained via MD modeling are employed as constitutive laws for both the materials and interfaces, as implemented in FE modeling. Three representative models are established covering the various possible material arrangements in wood cell walls. Under a 7-step loading protocol, all the models exhibit fixation, but only some models reveal a notable shape memory, where mechanical hotspots strengthen both fixation and recovery. SME elimination via removal of the moisture dependency of the interface clearly indicates the deterministic role of interface mechanics in the moistureinduced shape memory process. The shape memory steps are further explained from an energetics point of view. For any material exhibiting the SME phenomenon, there should be at least two substable states, corresponding to different moisture conditions in this study.

\section{Acknowledgements}

The authors acknowledge the support of the Swiss National Science Foundation (SNSF) (grant number 162957). S.K. acknowledges the support from an Office of Naval Research Director of Research Early Career Award (PECASE) (award No. N00014163175). C.Z. acknowledges Dr. Benoit Coasne for the insightful discussions.

\section{Author details}

${ }^{1}$ Department of Mechanical and Process Engineering, ETH Zurich, 8092 Zurich, Switzerland. ${ }^{2}$ Department of Civil and Environmental Engineering, Northwestern University, 2145 Sheridan Road, Evanston, IL 60208-3109, USA. ${ }^{3}$ Department of Civil and Building Engineering, Université de Sherbrooke, Sherbrooke, QC J1K 2R1, Canada

\section{Author contributions}

Conceptualization: C.Z., D.D., and J.C. Methodology: C.Z., S.K., D.D., and J.C. Formal analysis: C.Z., M.C., S.K., D.D., and J.C. Investigation: C.Z., M.C., D.D., and J. C. Data curation: C.Z. Visualization: C.Z. Supervision: D.D. and J.C. Writingoriginal draft: C.Z. Writing—review and editing: C.Z., M.C., D.D., and J.C. Funding acquisition: D.D. and J.C.

\section{Conflict of interest}

The authors declare no competing interests.

\section{Publisher's note}

Springer Nature remains neutral with regard to jurisdictional claims in published maps and institutional affiliations.

Supplementary information The online version contains supplementary material available at https://doi.org/10.1038/s41427-021-00342-8.

Received: 21 April 2021 Revised: 9 September 2021 Accepted: 24 September 2021.

Published online: 26 November 2021

\section{References}

1. Saifouni, O., Destrebecq, J.F., Froidevaux, J. \& Navi, P. Experimental study of the mechanosorptive behaviour of softwood in relaxation. Wood Sci. Technol. $\mathbf{5 0}$ 789-805 (2016).

2. Hajihassani, R., Mohebby, B., Najafi, S. K. \& Navi, P. Influence of combined hygro-thermo-mechanical treatment on technical characteristics of poplar wood. Maderas. Cienc. Tecnol. 0-0 (2018) https://doi.org/10.4067/S0718$221 \times 2018005011001$

3. Laine, K, Segerholm, K., Wålinder, M., Rautkari, L. \& Hughes, M. Wood densification and thermal modification: hardness, set-recovery and micromorphology. Wood Sci. Technol. 50, 883-894 (2016).

4. Navi, P. \& Pizzi, A. Property changes in thermo-hydro-mechanical processing. Holzforschung 69, 863-873 (2015).

5. Plaza, N., Zelinka, S. L., Stone, D. S. \& Jakes, J. E. Plant-based torsional actuator with memory. Smart Mater. Struct. 22, 072001 (2013).

6. Ugolev, B. N. Wood as a natural smart material. Wood Sci. Technol. 48, 553-568 (2014).

7. Derome, D., Rafsanjani, A., Patera, A., Guyer, R. \& Carmeliet, J. Hygromorphic behaviour of cellular material: hysteretic swelling and shrinkage of wood probed by phase contrast X-ray tomography. Philos. Mag. 92, 3680-3698 (2012).

8. Jakes, J. E. et al. Wood as inspiration for new stimuli-responsive structures and materials. Lakhtakia, A. In Proc. SPIE-International Society for Optical Engineering 9055, 90550K (13 pp.) (2014).

9. Navi, P. \& Heger, F. Combined densification and thermo-hydro-mechanical processing of wood. MRS Bull. 29, 332-336 (2004).

10. Dawson, C., Vincent, J. F. V. \& Rocca, A.-M. How pine cones open. Nature $\mathbf{3 9 0}$ 668-668 (1997).

11. Elbaum, R., Gorb, S. \& Fratzl, P. Structures in the cell wall that enable hygroscopic movement of wheat awns. J. Struct. Biol. 164, 101-107 (2008).

12. Colmars, J., Dubois, F. \& Gril, J. One-dimensional discrete formulation of a hygrolock model for wood hygromechanics. Mech. Time-Depend. Mater. 18, 309-328 (2014).

13. Behl, M. et al. Shape-memory polymers. Mater. Today 10, 20-28 (2010).

14. Chen, H.M., Wang, L. \& Zhou, S.B. Recent progress in shape memory polymers for biomedical applications. Chin. J. Polym. Sci. 36, 905-917 (2018).

15. S. Stevanic, J. \& Salmén, L. Molecular origin of mechano-sorptive creep in cellulosic fibres. Carbohydr. Polym. 230, 115615 (2020).

16. Xiao, C., Zhang, T., Zheng, Y., Cosgrove, D. J. \& Anderson, C. T. Xyloglucan deficiency disrupts microtubule stability and cellulose biosynthesis in 
Arabidopsis, altering cell growth and morphogenesis. Plant Physiol. 170, 234-249 (2016).

17. Park, Y. B. \& Cosgrove, D. J. Changes in cell wall biomechanical properties in the xyloglucan-deficient xxt1/xxt2 mutant of Arabidopsis. Plant Physiol. 158, 465-475 (2012).

18. Zhang, T., Zheng, Y. \& Cosgrove, D. J. Spatial organization of cellulose microfibrils and matrix polysaccharides in primary plant cell walls as imaged by multichannel atomic force microscopy. Plant J. 85, 179-192 (2016).

19. Zhang, T., Mahgsoudy-Louyeh, S., Tittmann, B. \& Cosgrove, D. J. Visualization of the nanoscale pattern of recently-deposited cellulose microfibrils and matrix materials in never-dried primary walls of the onion epidermis. Cellulose 21, 853-862 (2014).

20. Terrett, O. M. et al. Molecular architecture of softwood revealed by solid-state NMR. Nat. Commun. 10, 4978 (2019).

21. Da Silva, A. \& Kyriakides, S. Compressive response and failure of balsa wood. Int. J. Solids Struct. 44, 8685-8717 (2007).

22. Huang, R., Becker, A. A. \& Jones, I. A. Modelling cell wall growth using a fibrereinforced hyperelastic-viscoplastic constitutive law. J. Mech. Phys. Solids 60, 750-783 (2012).

23. Fahlén, J. \& Salmén, L. On the lamellar structure of the tracheid cell wall. Plant Biol. 4, 339-345 (2002).

24. Keplinger, T. et al. A zoom into the nanoscale texture of secondary cell walls. Plant Methods 10, 1 (2014).

25. Casdorff, K, Keplinger, T., Rüggeberg, M. \& Burgert, I. A close-up view of the wood cell wall ultrastructure and its mechanics at different cutting angles by atomic force microscopy. Planta 247, 1123-1132 (2018).

26. Salmén, L. \& Fahlén, J. Reflections on the ultrastructure of softwood fibers. Cellul. Chem. Technol. 40, 181-185 (2006).

27. Guo, J., Du, W., Wang, S., Yin, Y. \& Gao, Y. Cellulose nanocrystals: a layered host candidate for fabricating intercalated nanocomposites. Carbohydr. Polym. 157, 79-85 (2017).

28. Agarwal, U. P. Raman imaging to investigate ultrastructure and composition of plant cell walls: distribution of lignin and cellulose in black spruce wood (Picea mariana). Planta 224, 1141-1153 (2006).

29. Cosgrove, D. J. Re-constructing our models of cellulose and primary cell wall assembly. Curr. Opin. Plant Biol. 22, 122-131 (2014).

30. Zhang, C., Keten, S., Derome, D. \& Carmeliet, J. Hydrogen bonds dominated frictional stick-slip of cellulose nanocrystals. Carbohydr. Polym. 258, 117682 (2021).
31. Zhang, C. et al. Hygromechanical mechanisms of wood cell wall revealed by molecular modeling and mixture rule analysis. Sci. Adv. 7, (2021).

32. Zhang, C., Coasne, B., Guyer, R., Derome, D. \& Carmeliet, J. Moisture-induced crossover in the thermodynamic and mechanical response of hydrophilic biopolymer. Cellulose 27, 89-99 (2020).

33. Zhang, C. et al. Disentangling heat and moisture effects on biopolymer mechanics. Macromolecules 53, 1527-1535 (2020).

34. Derome, D., Kulasinski, K., Zhang, C., Chen, M. \& Carmeliet, J. Geitmann, A. \& Gril, J. in Plant Biomechanics 247-269 (Springer International Publishing, 2018).

35. Kulasinski, K., Salmén, L., Derome, D. \& Carmeliet, J. Moisture adsorption of glucomannan and xylan hemicelluloses. Cellulose 23, 1629-1637 (2016).

36. Kulasinski, K., Guyer, R., Keten, S., Derome, D. \& Carmeliet, J. Impact of moisture adsorption on structure and physical properties of amorphous biopolymers. Macromolecules 48, 2793-2800 (2015).

37. Frey-Wyssling, A. The fine structure of cellulose microfibrils. Science (80-) $\mathbf{1 1 9}$ 80-82 (1954).

38. Belbekhouche, S. et al. Water sorption behavior and gas barrier properties of cellulose whiskers and microfibrils films. Carbohydr. Polym. 83, 1740-1748 (2011).

39. Kulasinski, K., Keten, S., Churakov, S. V., Derome, D. \& Carmeliet, J. A comparative molecular dynamics study of crystalline, paracrystalline and amorphous states of cellulose. Cellulose 21, 1103-1116 (2014).

40. Mark, R. E. Cell Wall Mechanics of Tracheids (Yale University Press, 1967).

41. Nakamura, K, Wada, M., Kuga, S. \& Okano, T. Poisson's ratio of cellulose la and cellulose II. J. Polym. Sci. Part B 42, 1206-1211 (2004).

42. Chen, L, Li, A. He, X. \& Han, L. A multi-scale biomechanical model based on the physiological structure and lignocellulose components of wheat straw. Carbohydr. Polym. 133, 135-143 (2015).

43. Zhang, C. et al. Hygromechanics of softwood cellulosic nanocomposite with intermolecular interactions at fiber-matrix interface investigated with molecular dynamics. Compos. Part B Eng. 228, 109449 (2021).

44. Ghobadi, E., Heuchel, M., Kratz, K. \& Lendlein, A. Atomistic simulation of the shape-memory effect in dry and waterswollen poly[(rac-lactide)-co-glycolide] and copolyester urethanes thereof. Macromol. Chem. Phys 215, 65-75 (2014).

45. McNaught, A. \& Wilkinson, A. The IUPAC Compendium of Chemical Terminology (International Union of Pure and Applied Chemistry (IUPAC), 2019).

46. Carmeliet, J., Derome, D., Dressler, M. \& Guyer, R. A. Nonlinear poro-elastic model for unsaturated porous solids. J. Appl. Mech. 80, 020909 (2013). 